Supporting Information

Design of In-situ Poled $\mathrm{Ce}^{3+}$ Doped Electrospun PVDF/Graphene Composite Nanofibers for Fabrication of Nano-pressure Sensor and Ultrasensitive Acoustic Nanogenerator

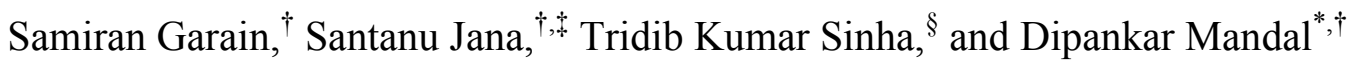

${ }^{\dagger}$ Organic Nano-Piezoelectric Device Laboratory, Department of Physics, Jadavpur University, Kolkata 700032, India

Department of Electronics, Netaji Nagar Day College, 170/436 N. S. C Bose Road, Kolkata 700092, India

${ }^{\S}$ Materials Science Centre, Indian Institute of Technology (IIT), Kharagpur 721302, India

*Corresponding Author

Dr. Dipankar Mandal

E-mail:dipankar@phys.jdvu.ac.in 


\section{S1. Raman Spectroscopy of Graphene}

Raman spectroscopy is a fast and non-destructive method for the characterization of carbons. ${ }^{\mathrm{S} 1}$ The spectra show common features in the $1200-3000 \mathrm{~cm}^{-1}$ region: the $\mathrm{G}$ and D peaks, which lie at around 1560 and $1360 \mathrm{~cm}^{-1}$ respectively (Figure S1). The G peak corresponds to the $\mathrm{E}_{2 \mathrm{~g}}$ phonon at the Brillouin zone center. The D peak is due to the breathing modes of $\mathrm{sp}^{2}$ atoms and requires a defect for its activation. ${ }^{\mathrm{S} 2 \mathrm{~S} 3}$ The D peak intensity is not related to the number of graphene layers, but only to the amount of disorder. ${ }^{\mathrm{S} 2}$ The $2 \mathrm{D}$ peak is the second order of the $\mathrm{D}$ peak. This is a single peak in monolayer graphene, whereas it splits in multi-layer graphene, reflecting the evolution of the band structure (Figure S1). ${ }^{\mathrm{S} 1}$ The 2D peak is always seen, even when no D peak is present, since no defects are required for the activation of two phonons with the same momentum. ${ }^{\mathrm{S} 2}$

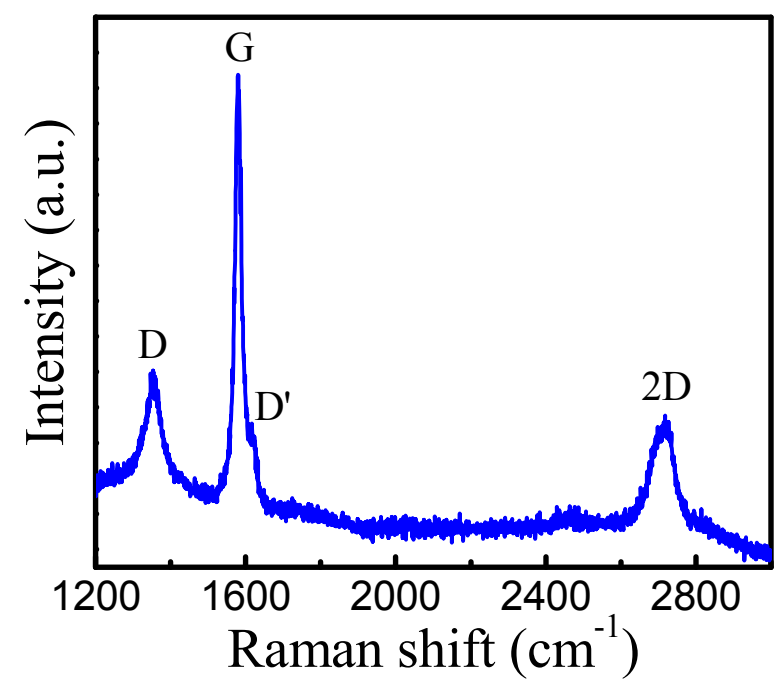

Figure S1. Raman spectrum with a $514 \mathrm{~nm}$ excitation laser wavelength of as prepared graphene.

The spectra having small D band compare to the $\mathrm{G}$ band, indicating an absence of defects (Figure S1). It is possible to identify the number of layers from the shape of the 2D peak. ${ }^{\mathrm{S} 1, \mathrm{~S} 3}$ 
Ferrari et al., reported that for single layer graphene the 2D band is observed to be a single symmetric peak with a full width at half maximum (FWHM) of $\sim 30 \mathrm{~cm}^{-1}$. S2 Adding successive layers of graphene causes the 2D band to split into several overlapping modes and the resulting FWHM of $\sim 73 \mathrm{~cm}^{-1}$ (Figure S1). The band splitting of the 2D band going from single layer graphene to multilayer graphene arises from symmetry lowering that takes place when increasing the layers of graphene in the sample.

\section{S2. Calculation of Crystallinity}

The total degree of total crystallinity $\left(\chi_{c_{t}}\right)$, $\beta$-crystallinity $\left(\chi_{c_{\beta}}\right)$ and $\gamma$-crystallinity $\left(\chi_{c_{\gamma}}\right)$ are calculated by the following equations

$$
\begin{aligned}
& \chi_{\mathrm{c}_{\mathrm{t}}}=\frac{\sum A_{c r}}{\sum A_{c r}+\sum A_{a m r}} \times 100 \% \\
& \chi_{\mathrm{c}_{\beta}}=\chi_{\mathrm{c}_{\mathrm{t}}} \times \frac{\sum A_{\beta}}{\sum A_{\beta}+\sum A_{\gamma}} \% \\
& \chi_{\mathrm{c}_{\gamma}}=\chi_{\mathrm{c}_{\mathrm{t}}} \times \frac{\sum A_{\gamma}}{\sum A_{\beta}+\sum A_{\gamma}} \%
\end{aligned}
$$

where, $\sum A_{c r}$ and $\Sigma A_{a m r}$ are the summation of the integral area of the crystalline peaks and the amorphous halo from PVDF respectively. $\sum A_{\beta}$ and $\sum A_{\gamma}$ indicate the total integral area from $\beta$ and $\gamma$-crystalline phases peaks respectively.

\section{S3. Calculation of $\boldsymbol{F}_{E A \text {. }}$}

For a system containing $\alpha$-, $\beta$-, and $\gamma$-phases the relative electroactive phase fraction $\left(F_{E A}\right.$ including $\beta$ - and $\gamma$-phases) can be calculated using the following equation.

$$
F_{\mathrm{EA}}=\frac{A_{E A}}{\left(\frac{K_{841}}{K_{764}}\right) A_{764}+A_{E A}} \times 100 \%
$$


where $A_{E A}$ and $A_{764}$ are the absorbance intensities at 841 and $764 \mathrm{~cm}^{-1}$, respectively, and $\mathrm{K}_{841}$ $\left(7.7 \times 104 \mathrm{~cm}^{2} \mathrm{~mol}^{-1}\right)$ and $\mathrm{K}_{764}\left(6.1 \times 104 \mathrm{~cm}^{2} \mathrm{~mol}^{-1}\right)$ are the absorption coefficients at the respective wavenumbers.

\section{S4. Input pressure $(\sigma)$ calculation}

The calculation of the pressure input induced by finger impact and thermocol (polystyrene foamed) falling were based on the physical model combining the gravity and pulse term. ${ }^{\mathrm{S} 4}$ Therefore, based on the kinetic energy and momentum theorem, we have calculated based on the following basic equations:

$$
\begin{aligned}
& \mathrm{m} \cdot \mathrm{g} \cdot \mathrm{h}=\frac{1}{2} \mathrm{mv}^{2} \\
& (\mathrm{~F}-\mathrm{m} \cdot \mathrm{g}) \cdot \Delta \mathrm{t}=\mathrm{m} \cdot \mathrm{v} \\
& \sigma=\frac{F}{\mathrm{~S}}
\end{aligned}
$$

Here, $\mathrm{m}$ is the mass of the object, $\mathrm{g}=9.8 \mathrm{~N} / \mathrm{kg}$, $\mathrm{h}$ is the falling height, $\mathrm{v}$ is the maximum falling velocity, $\sigma$ is the contact pressure or applied stress, $\mathrm{F}$ is the contact force, $\mathrm{S}$ is the contact area, and $\Delta t$ is the time spanning during the process. 


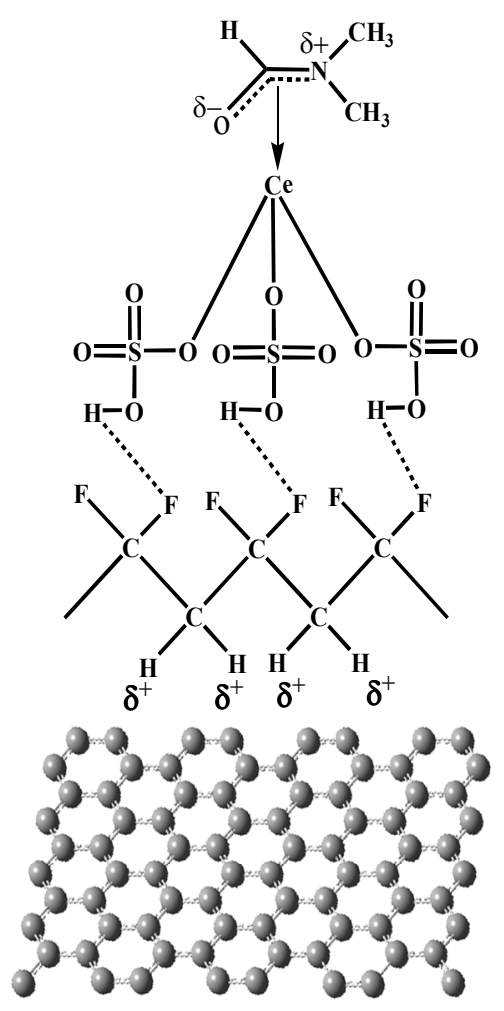

Figure S2. Formation of $\mathrm{H}$-bonding with $-\mathrm{HSO}_{4}$ and $\mathrm{CF}_{2}$ dipoles of PVDF. Interaction between the $\beta$-phase of PVDF and graphene sheet.

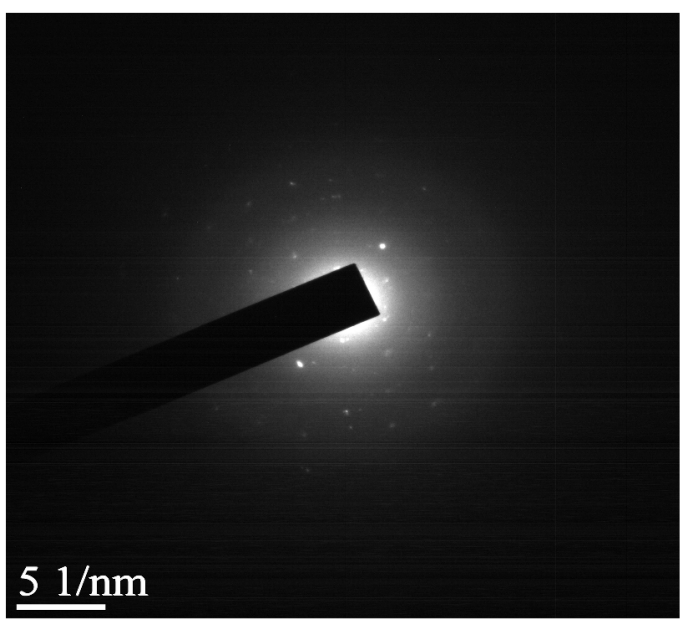

Figure S3. SAED pattern of the PVDF-Ce-G NFs. 


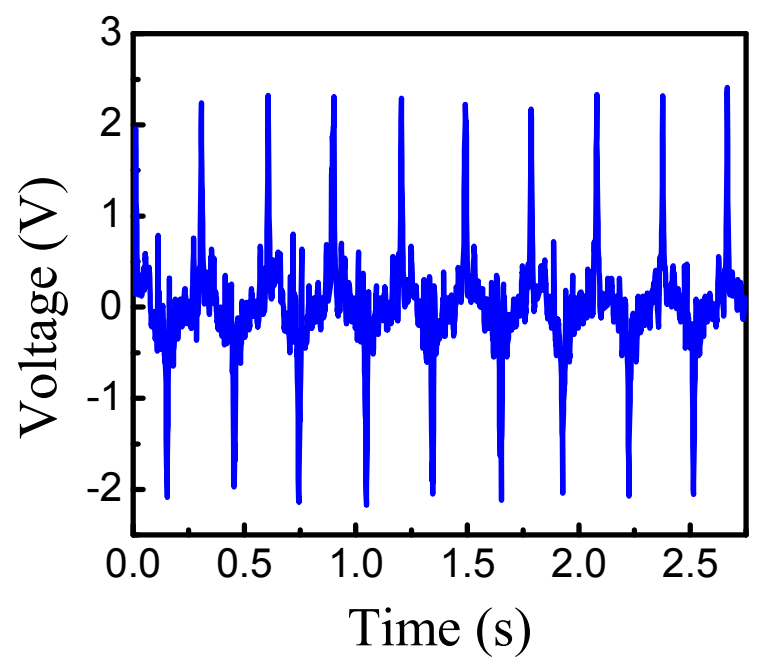

Figure S4. Output voltage response due to squeeze the ANG laterally by human finger.

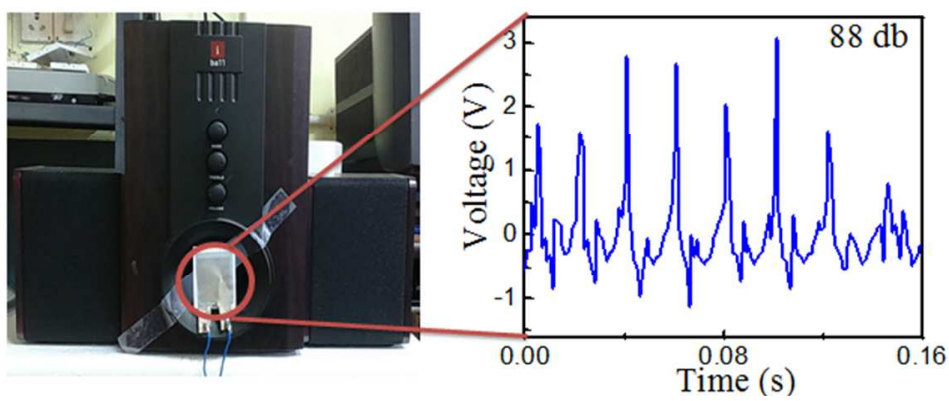

Figure S5. Open circuit output voltage response from the ANG driven by sound vibration from speaker. 


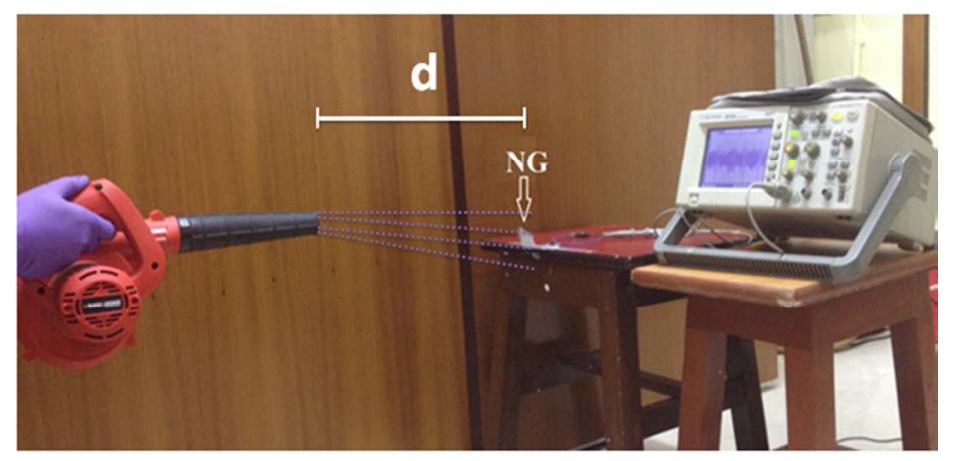

Figure S6. Photograph of the experimental setup to study the response from NG under the variable air flow velocity.

\section{REFERENCES}

S1. Ferrari, A. C.; Meyer, J. C.; Scardaci, V.; Casiraghi, C.; Lazzeri, M.; Mauri, F.; Piscanec, S.; Jiang, D.; Novoselov, K. S.; Roth, S.; Geim, A. K. Raman Spectrum of Graphene and Graphene Layers. Phys. Rev. Lett. 2006, 97, 187401-187404.

S2. Ferrari, A. C. Raman Spectroscopy of Graphene and Graphite: Disorder, Electron-phonon Coupling, Doping and Nonadiabatic Effects. Solid State Commun. 2007, 143, 47-57.

S3. Dresselhaus, M. S.; Jorio, A.; Hofmann, M.; Dresselhaus, G.; Saito, R. Perspectives on Carbon Nanotubes and Graphene Raman Spectroscopy. Nano Lett. 2010, 10, 751-758.

S4. Hou, C.; Huang, T.; Wang, H.; Yu, H.; Zhang, Q.; Li, Y. A Strong and Stretchable Selfhealing Film with Self-activated Pressure Sensitivity for Potential Artificial Skin Applications. Sci. Rep. 2013, 3, 3138-3142. 